\title{
Electrochemotherapy with bleomycin of different types of cutaneous tumours in a ferret (Mustela putorius furo)
}

\author{
Jozko Racnik¹, Tanja Svara², Marko Zadravec¹, Mitja Gombac², Maja Cemazar³, \\ Gregor Sersa ${ }^{3}$, Natasa Tozon ${ }^{4}$
}

\begin{abstract}
${ }^{1}$ Clinic for Birds, Small Mammals and Reptiles, Institute of Poultry, Birds, Small Mammals and Reptiles, Veterinary Faculty, University of Ljubljana, Ljubljana, Slovenia

${ }^{2}$ Institute of Pathology, Wild Animals, Fish and Bees, Veterinary Faculty, University of Ljubljana, Ljubljana, Slovenia

${ }^{3}$ Institute of Oncology Ljubljana, Ljubljana, Slovenia

${ }^{4}$ Small Animal Clinic, Veterinary Faculty, University of Ljubljana, Ljubljana, Slovenia
\end{abstract}

Radiol Oncol 2018; 52(1): 98-104.

Received 3 October 2017

Accepted 5 November 2017

Correspondence to: Jozko Racnik, Ph.D., Clinic for Birds, Small Mammals and Reptiles, Institute of Poultry, Birds, Small Mammals and Reptiles, Veterinary Faculty, University of Ljubljana, Cesta v Mestni log 47, SI-1000 Ljubljana, Slovenia; E-mail: josko.racnik@vf.uni-lj.si

Disclosure: no potential conflicts of interest were disclosed.

Background. Mast cell tumour, sebaceous gland adenoma, and less common squamous papilloma are skin tumours in ferrets (Mustela putorius furo), and early excisional surgery is usually the treatment of choice. The aim of our study was to investigate the effectiveness of electrochemotherapy (ECT), a new, minimally invasive non-surgical method, as first treatment option of different types of ferret skin tumours located on surgically difficult sites.

Materials and methods. A 5-year-old castrated male ferret with two cutaneous masses, presenting 4 months apart and a 7-year-old spayed female ferret with two cutaneous masses, that appeared simultaneously on two locations are presented. In the first patient, both masses were diagnosed as mast cell tumours, and in the second patient, squamous papilloma and sebaceous adenoma were diagnosed. One session of ECT with bleomycin injected intratumourally was applied in all tumours.

Results. Complete response (CR) of all tumours was obtained, without recurrence during observation period of 15 months after ECT for first tumour and 11 months after ECT of the tumour located on the right hock in first patient, and 8 months after treatment for the second patient.

Conclusions. In present study, ECT with bleomycin proved to be safe and effective against different cutaneous tumours in ferrets. Due of good results, low cost and relatively easy procedure, ECT could be the treatment of choice instead of surgery for the selected skin tumours in ferrets.

Key words: electrochemotherapy; bleomycin; cutaneous tumours, ferret

\section{Introduction}

Tumours are common health problem in domestic pet ferrets (Mustela putorius furo), most commonly affected are endocrine, integumentary and lymphatic system. ${ }^{1}$ Mast cell tumours (MCTs) and sebaceous tumours (sebaceous epitelioma, sebaceous adenoma) are most prevalent tumours of the skin. ${ }^{2}$

Most frequently, MCTs appear on the extremities, following the trunk and head or neck. They are usually small (1-4 $\mathrm{mm}$ in diameter), round to plaque-like nodules with surface crusting. ${ }^{3}$ In some cases, animals develop local pruritus, and overlying skin may be ulcerated due to self-trauma. ${ }^{1}$ In ferrets, MCTs are considered clinically benign and they do not spread locally or metastasize. ${ }^{1,3}$ However, there are some suggestions about visceral involvement and malignant behaviour of this tumours. ${ }^{4}$

Sebaceous adenomas are benign tumours, which involve only skin and may occur anywhere on the 


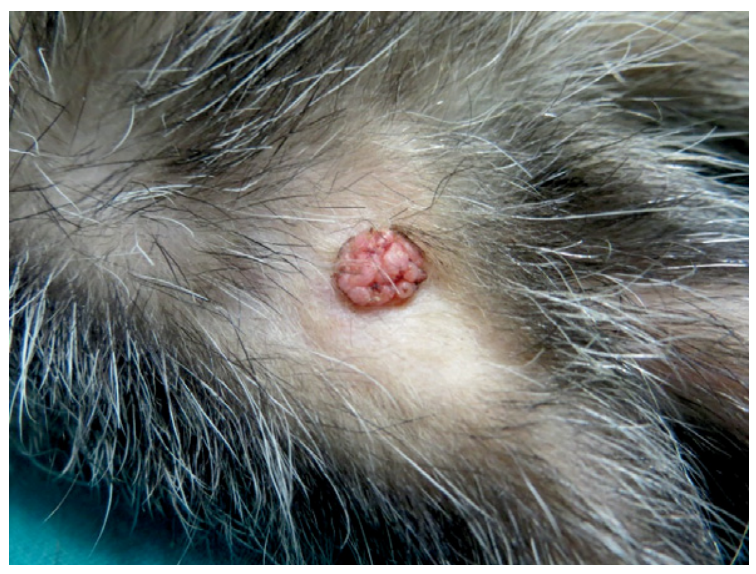

FIGURE 1. Macroscopic presentation of squamous papilloma (Tumour 4).

body. They are firm, warty and often multinodular, sometimes looking very similar to MCTs. They can be ulcerated, irritated and traumatised with local inflammation., ${ }^{2,4}$

Squamous papilloma is very rare skin tumour of ferrets with only one case noted in a study of 1,525 ferret neoplasia's collected in years 1990 to $2000 .^{5}$ Although there is some evidence that some squamous cell carcinomas (SCC) of the skin in ferrets can be associated with papillomavirus ${ }^{6}$, possible viral aetiology of cutaneous squamous papilloma in ferrets was yet not investigated. Moreover, recent publications suggested that, squamous papilloma of skin and conjunctiva in dogs are not connected with papillomaviruses. ${ }^{7}$

Fine needle aspiration (FNA), incisional or excisional biopsy are recommended to confirm the diagnosis of any cutaneous tumour. ${ }^{2}$ Early excisional surgery is the treatment of choice, and wide

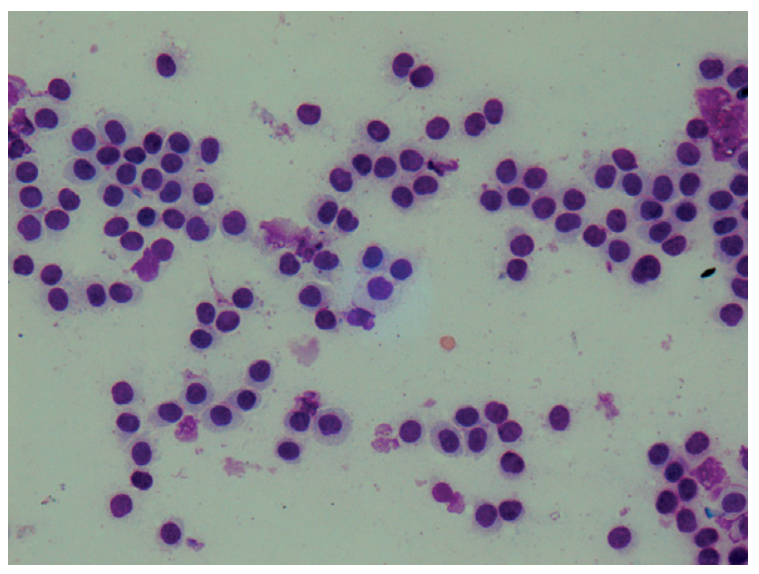

FIGURE 2. Cytological presentation of mast cell tumours (Tumour 2). Hemacolor, $400 \mathrm{x}$. surgical margins are always advisable., ${ }^{2,5}$ However, some tumours are large with invading surrounding tissue on unsuitable sites, which makes the closure of defect after surgery, and consequently preservation of function, difficult. ECT is a new anti-neoplastic therapy in veterinary medicine that combines administration of a chemotherapeutic agent, cisplatin or bleomycin, with electric pulses to the tumour. ${ }^{8}$

The aim of our study was to investigate the effectiveness of electrochemotherapy (ECT), as a new, minimally invasive non-surgical method, for treatment of different types of ferret skin tumours located on surgically difficult sites.

\section{Materials and methods}

\section{Patients}

First patient was a 5-year-old castrated male ferret with two cutaneous masses presented 4 months apart. First mass $(0.8 \mathrm{~cm} \times 0.8 \mathrm{~cm} \times 0.5 \mathrm{~cm})$ was located on the laterodorsal tail base (tumour 1), while the second one $(1.2 \mathrm{~cm} \times 1.0 \mathrm{~cm} 0.7 \mathrm{~cm})$ was found on the lateral side of the right hock (tumour 2). The ferret had no major health issues since the initial presentation, and masses apparently did not produce any health problems or discomfort. Regular health checks and vaccinations were carried out every year.

Second patient was a 7-year-old spayed female ferret with two cutaneous masses that appeared simultaneously on two locations. First mass (0.7 $\mathrm{cm} \times 0.7 \mathrm{~cm} \times 0.5 \mathrm{~cm}$ ) was located on the lateral side of the left hock (tumour 3) (Figure 1), while the location of the second mass $(0.4 \mathrm{~cm} \times 0.4 \mathrm{~cm}$ $x 0.2 \mathrm{~cm}$ ) was dorsolateral on the left chest region (tumour 4). Before the initial presentation, the ferret had a history of insulinoma managed with diet, corticosteroids and regular glucose measurements. On physical examination, both patients were alert, bright and responsive with body masses of $1700 \mathrm{~g}$ and $430 \mathrm{~g}$ respectively. Incisional biopsies of tumours 1, 3 and 4, and FNA of tumour 2 were obtained under general gaseous anaesthesia, and histopathology and cytology were done. Briefly, samples for histopathology were fixed in 10\% buffered formaline and routinely embedded in paraffin. Four- $\mu \mathrm{m}$-thick sections were stained with hematoxylin and eosin and examined microscopically. Cytological smears were stained with Hemacolor (Merck ${ }^{\circledR}$, Darmstadt, Germany).

Histopathological examination of the tumour 1 presented as nonencapsulated, slightly infiltrative 


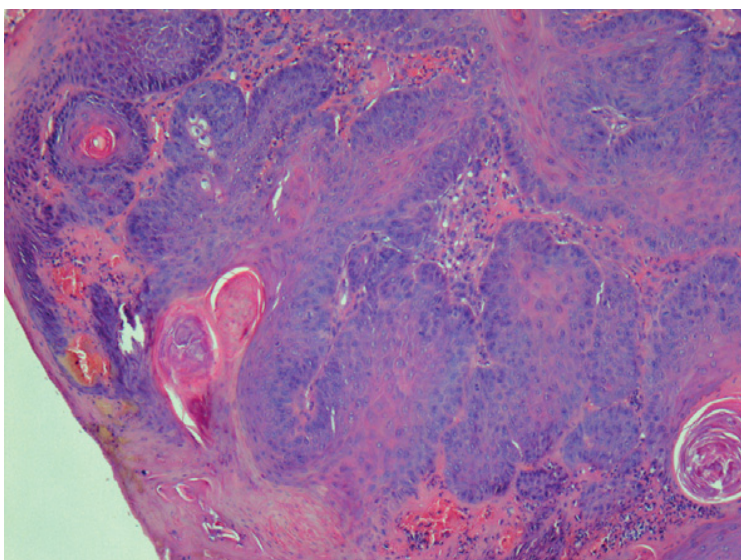

FIGURE 3. Histological presentation of squamous papilloma (Tumour 4). Hematoxylin and eosin stain, $100 \mathrm{x}$.

tumour composed of round cells that exhibited mild anisocytosis. Neoplastic cells had a moderate amount of pale blue cytoplasm and round nuclei that exhibited mild anisokaryosis. Additional Toluidine blue staining revealed numerous cytoplasmic metachromatic granules. Mitotic figures were rare.

Cytological samples of the tumour 2 (Figure 2) contained moderate number of the neoplastic cells with the morphology similar to neoplastic cells in the tumour 1 . Single eosinophils were scattered between neoplastic cells.

Histopathology of tumour 3 (Figure 3) exhibited epithelial tumour that formed papillary projections supported by a fine to moderate fibrovascular core. Papillary projections were covered with welldifferentiated squamous epithelial cells. Changes characteristic for papilloma, such as enlarged keratohyalin granules, koilocytes and intranuclear inclusions, were not present. Mitotic figures were 6 per 10 high power fields (HPF). The stroma was diffusely moderately infiltrated with neutrophils.

Histopathology of tumour 4 exhibited lobular tumour that consisted predominantly of cells with sebaceous differentiation (sebocytes) and peripheral layer of basaloid reserve cells. Sebocytes were round to oval, exhibited moderate anisocytosis and had finely vacuolated cytoplasm. Basaloid reserve cells were cuboid and had a small amount of basophilic cytoplasm, and round to oval nuclei. Mitotic figures were rare, 3 per $10 \mathrm{HPF}$, and confined to basaloid reserve cells.

In the first patient, both masses were diagnosed as MCTs, and in the second patient, squamous papilloma of hock region and sebaceous adenoma of chest region were diagnosed. Haematology,

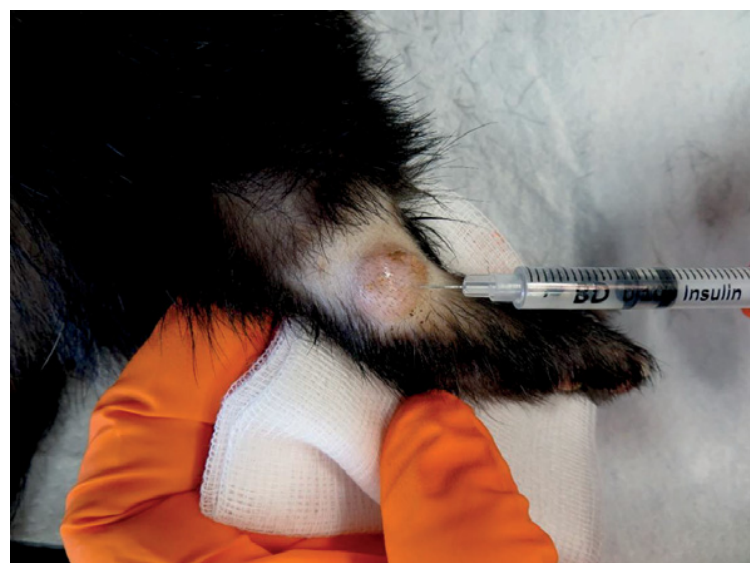

FIGURE 4. Intralessional injection of bleomycin into mast cell tumour (Tumour 2) located laterally on the right hock. Note the size of tumor and surgicaly chalenging anatomical location.

serum biochemistry, total body radiographs, and abdominal ultrasound were performed for staging. Whole body radiographs and abdominal ultrasound investigations showed no evidence of health problems or distant metastasis. In the first patient, the haematology showed no abnormal findings, most of serum biochemistry values were within reference range; only urea nitrogen $(12.4 \mathrm{mmol} / \mathrm{L}$; reference $\left.3.93-8.93 \mathrm{mmol} / \mathrm{L}^{9}\right)$ and creatinine $(128$ umol/L; reference $26.52-70.72 \mu \mathrm{mol} / \mathrm{L}^{9}$ ) were elevated suggesting early kidney disease. Also in the second patient, haematology results showed no abnormal findings; however, serum biochemistry showed elevated aspartate aminotransferase (248 $\mathrm{U} / \mathrm{L}$; reference $78-140 \mathrm{U} / \mathrm{L}^{9}$ ) and decreased values of glucose $(2.8 \mathrm{mmol} / \mathrm{L}$; reference $5.5-7.5 \mathrm{mmol} /$ $\mathrm{L}^{9}$ ), most probably due to insulinoma and chronic gastroenteritis. The treatment possibilities, including surgery, were discussed with the owner. Due to unsuitable locations for surgical treatment and contemporary morbidities, ECT was selected as the minimally invasive treatment option. The owners were thoroughly informed about the procedure and its possible side effects and signed informed consent for application of electrochemotherapy.

\section{Electrochemotherapy}

For the ECT, patients were anesthetised using isoflurane and $0.5 \mathrm{mg} \sim$ volume of $0.1 \mathrm{ml}$ of bleomycin $(3 \mathrm{mg} / \mathrm{ml})$ was injected intratumourally using 29 gauge needle (Figure 4, 5). After 1-2 minutes, eight electric pulses of $100 \mu$ duration with amplitude to electrode distance ratio of $1300 \mathrm{~V} / \mathrm{cm}$ and frequency of $5 \mathrm{kHz}$ were delivered using plate 


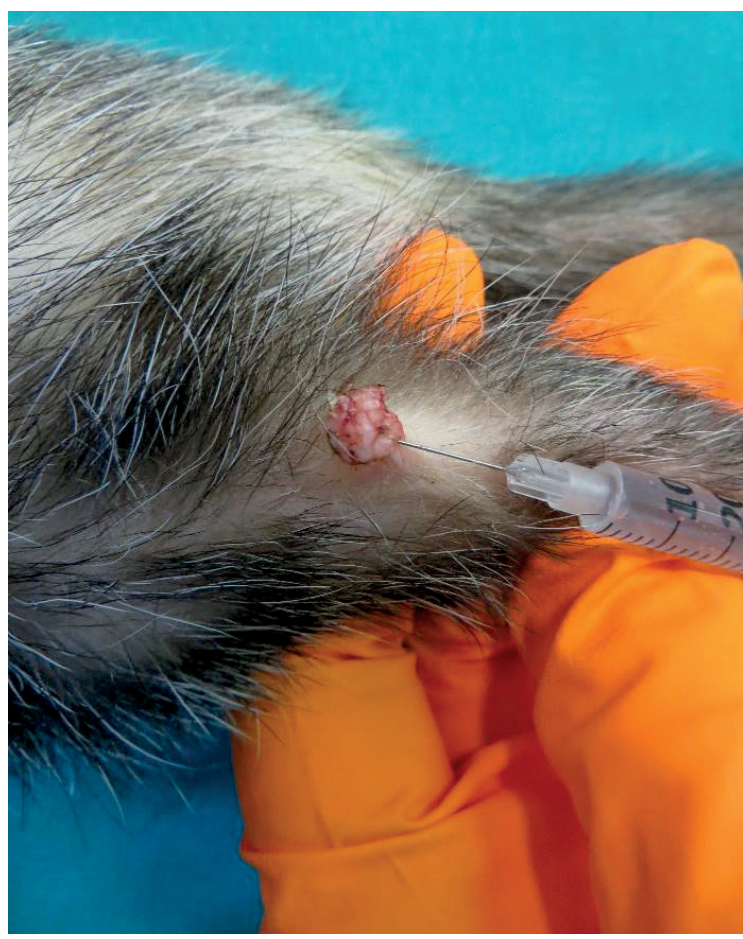

FIGURE 5. Intralessional injection of bleomycin into squamous papilloma (Tumour 4).

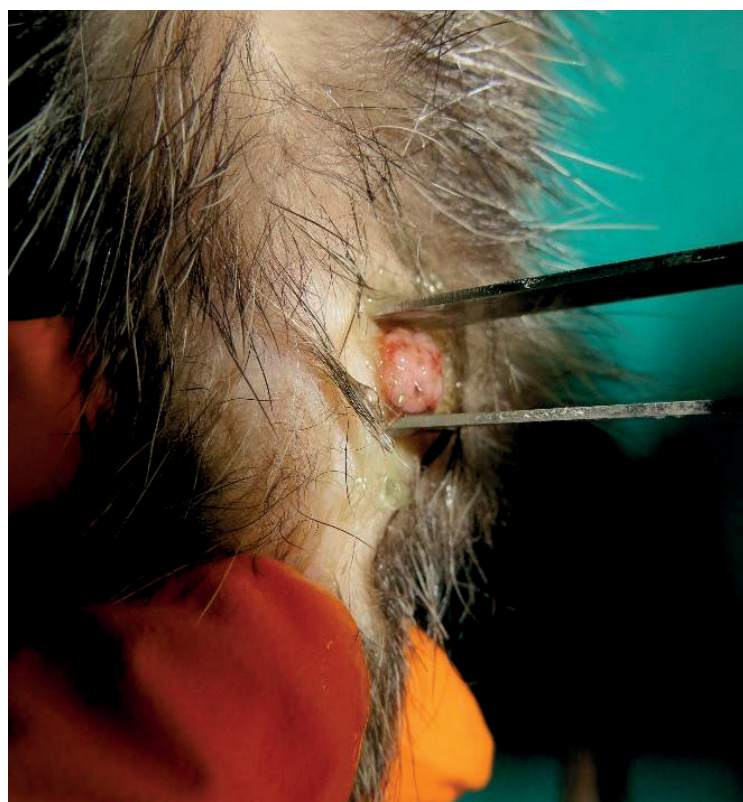

FIGURE 7. Electric pulses were delivered to the squamous papilloma (Tumour 4) using plate electordes.

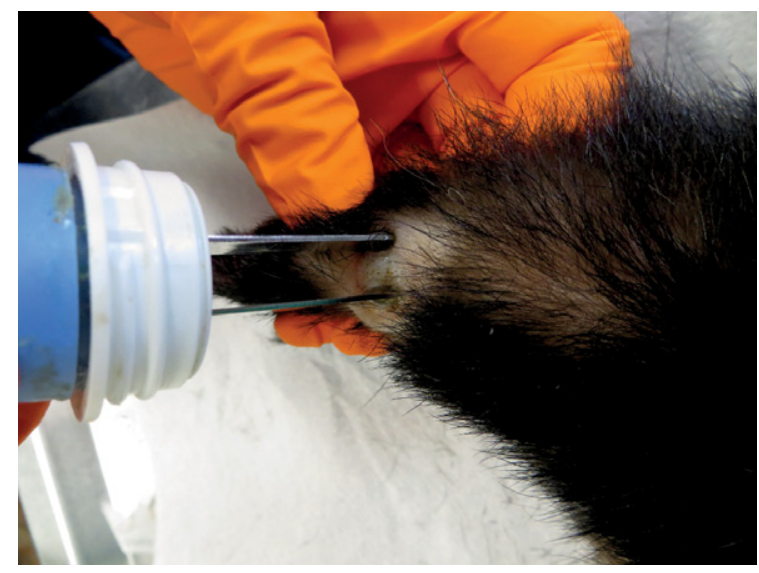

FIGURE 6. Electric pulses were delivered to the mast cell tumour (Tumour 2) using plate electordes.

electrodes (distance between the electrodes: $6 \mathrm{~mm}$ ) (Figures 6,7). Good contact between the electrodes and tumour mass was obtained by application of conductive gel to the treatment area. Electric pulses were generated by the electric pulse generator (Cliniporator $^{\mathrm{TM}}$ - IGEA srl, Carpi [MO], Italy). The entire tumour was exposed to electric pulses by moving the electrodes perpendicular through the whole volume of mass and the surrounding skin, starting on the tumour periphery. The duration of each ECT cycle was approximately 15 minutes, and in both cases, the recovery from anaesthesia was uneventful. Meloxicam (Loxicom; Norbrook Laboratories) in a dose $0.2 \mathrm{mg} / \mathrm{kg} /$ day $^{9}$ was prescribed for 3 consecutive days orally after every cycle of ECT to reduce pain and inflammation.

\section{Evaluation of response}

After the treatment, the patients were examined at regular intervals, depending on the owner's visit of clinic, to evaluate the treatment response. At each visit, the treated tumours were examined and photographed. In the meantime, the owner observed the treated area and reported the progress.

\section{Results}

No local or/and systemic side effects were noted during or after ECT. During ECT, some muscle contractions of the patients were observed after the application of each train of electric pulses. The contractions were mild, disappearing after the end of each train of electric pulses. All tumours became necrotic and regressed. In the first patient, 


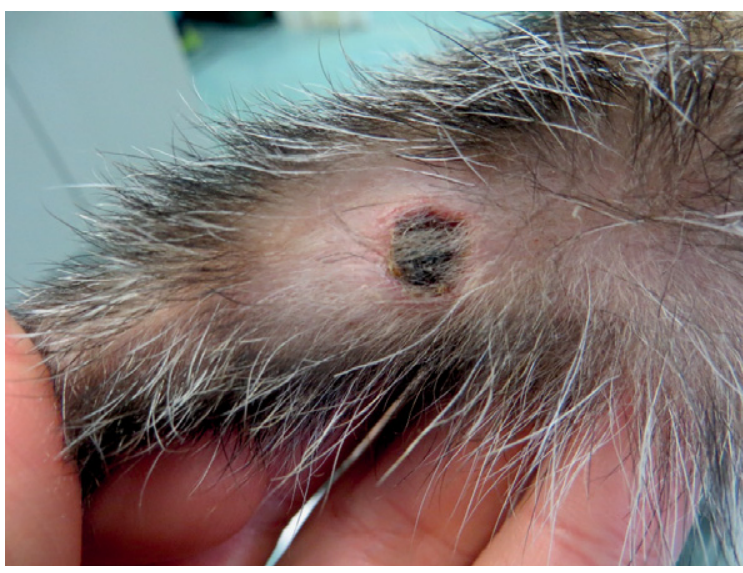

FIGURE 8. Necrosis with superficial scab of squamous papilloma (Tumour 4) 8 days after electrochemotherapy.

the MCT located on the laterodorsal tail base became necrotic on day 6 and a complete response (CR) was seen approximately 1 month after ECT, while the second MCT found on the lateral side of the right hock became necrotic on day 15 and CR was seen approximately two months (67 days) after ECT (Figure 9). In second patient, both tumours became necrotic on day 8 (Figure 8 ) and CRs were seen 70 days after ECT (Figure 10). In first patient, no tumour growth has been seen during the observation period of 15 months after ECT of the tumour located on the tail base, and 11 months after ECT of the tumour located on the right hock. In second patient, no tumour recurrence was detected 8 months after treatment.

\section{Discussion}

ECT with bleomycin as a single treatment was an effective treatment of cutaneous neoplasms; MCTs, sebaceous adenoma, and squamous papilloma in our cases. ECT was well tolerated by the patients and is a minimally invasive procedure with no major side effects noted, with excellent responses and long-lasting CR. In first patient, no tumour growth was seen during observation period of 15 months after ECT of the tumour located on the tail base, and 11 months after ECT of the tumour located on the right hock. In second patient, no tumour regrowth was seen 8 months after treatment. Different studies reported an excellent treatment response of ECT with cisplatin or bleomycin in tumours of different histology with minimal side effects in dogs ${ }^{10,11}$, cats $^{12}$, horses ${ }^{13}$, pet rats $^{14}$, and reptiles ${ }^{15,16}$. In our cases, only some muscle contractions of the

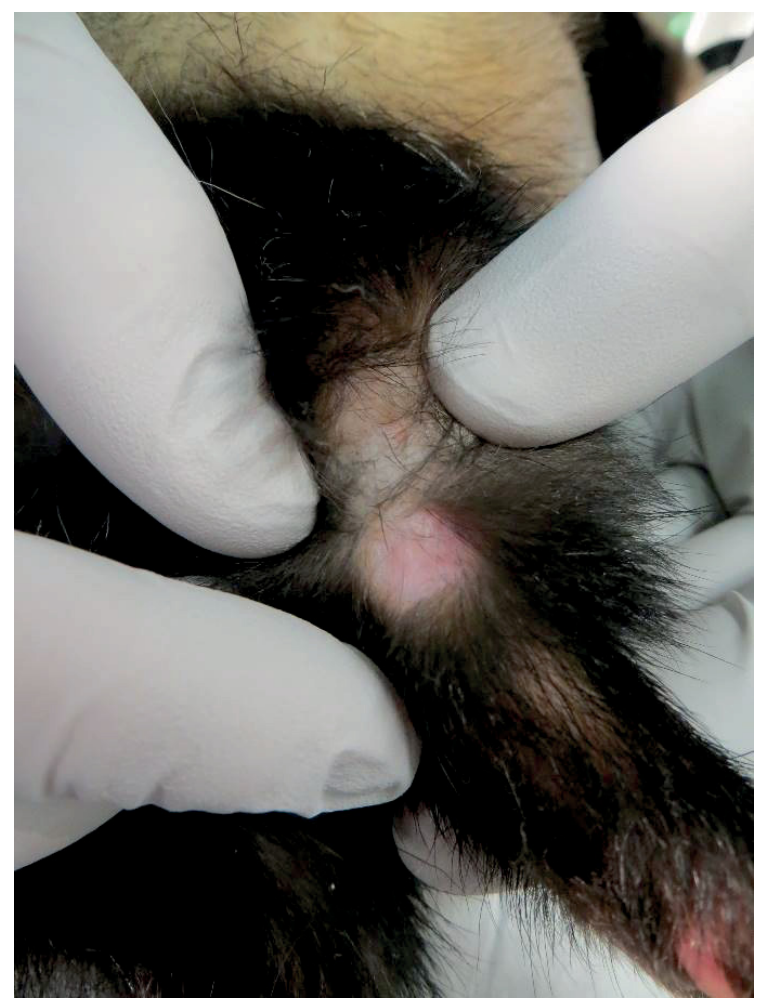

FIGURE 9. Complete response of mast cell tumour (Tumour 2) 67 days after electrochemotherapy.

patient were observed during the application of electric pulses. The contractions were mild, disappearing after the end of each train of electric pulses. In animals, pain during ECT can be avoided using sedation and/or general anaesthesia. ${ }^{10-16}$ Gaseous anaesthesia with isoflurane was used in our cases to prevent pain and facilitate proper position of patients for tumour manipulation, the application of chemotherapeutic agent, and positioning of electrodes. Meloxicam, a nonsteroidal anti-inflammatory analgesic was prescribed to the patients before and after ECT to reduce pain and inflammation. ${ }^{9}$ According to the literature, surgery with wide margins is advised as the first treatment option for benign skin tumours in ferrets 2,5 , but this therapeutic option is not always applicable due to anatomical locations. In author's experience, some skin tumours could be quite large and located on toe, hock or footpad. Thus, aggressive surgery could result in loss of physiological function of a body part and a non-favourable cosmetic effect of therapy. In both ferrets, the tumour locations were not optimally positioned for surgery, and complications could arise with the skin defect too big for adequate closure. In that context, new therapies like ECT are highly beneficial, because of good an- 


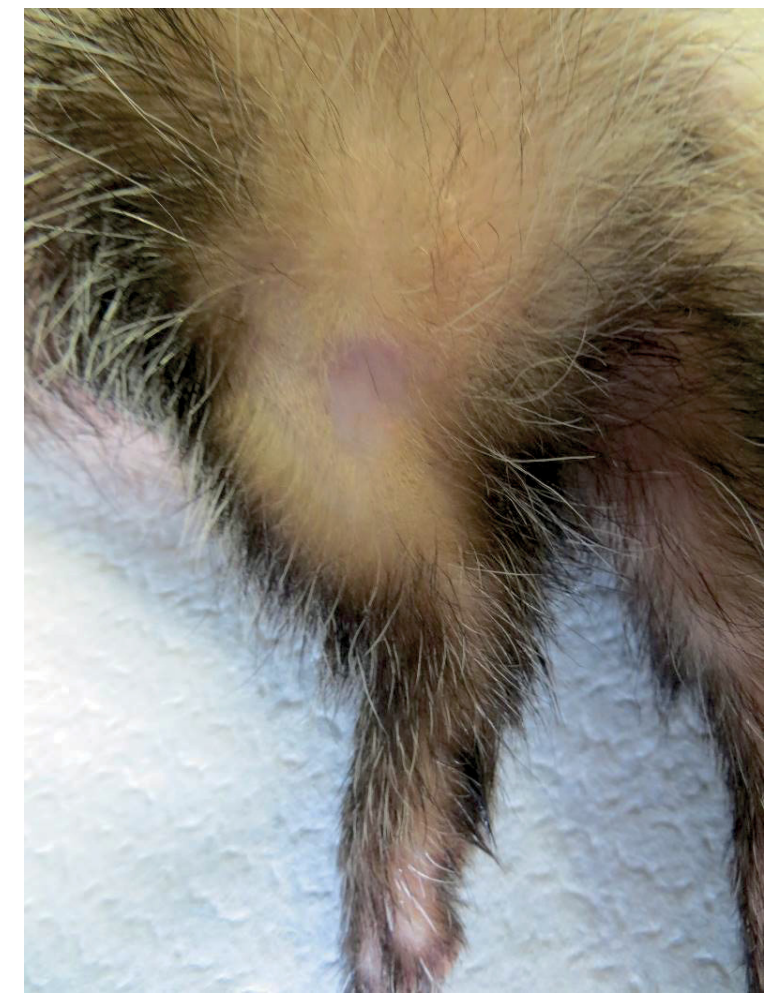

FIGURE 10. Complete response of squamous papilloma (Tumour 4) - 70 days after electrochemotherapy.

ti-tumour effectiveness, combined with excellent functional and cosmetic effects, while being less invasive than surgery. $8,10,11,12$

Neoplastic diseases are commonly seen in ferrets, and medical treatment of tumours with chemotherapy is well reported.1,17,18 Different chemotherapeutic agents are used in ferret oncology, including bleomycin. ${ }^{17,19}$ Bleomycin is an antitumour antibiotic commonly used in human and veterinary medicine. In exotic pets, bleomycin was used intralesionally in the treatment of SCC in a greater hedgehog tenrec (Setifer setosus) and in a blue fronted amazon parrot (Amazona aestiva) for xanthoma, respectively. ${ }^{17}$ Recently, ECT with bleomycin was reported in a yellow bellied slider (Trachemys scripta scripta) and in a green sea turtle (Chelonia mydas) for the treatment of SCC and fibropapilloma with excellent therapeutic outcome and minimal side effects. ${ }^{15,16,17}$ In ferret oncology, the data about this chemotherapeutic agent are scarce; however, bleomycin was used in the therapy of metastatic SCC in one animal at a dosage of $10-20 \mathrm{U} / \mathrm{m}^{2}$ once a week, injected subcutaneously with moderate antitumour effectiveness. ${ }^{19}$ In our patients, ECT using bleomycin did not induce any clinically evident systemic side effects; only mild, local tumour ne- crosis as a consequence of successful treatment was seen in the week following the treatment in both cases without notable effect on the patient's condition. The superficial scab developed after necrosis; it fell off within few weeks after therapy.

The drawback of our study is that it presents the ECT treatment effectiveness on only two patients. For broader acceptance of ECT in treatment of tumours in ferrets larger cohort of patients and higher number of tumours is needed. Though this study as the first provides evidence of safety and effectiveness of ECT in this specific animal species.

In conclusion, ECT with bleomycin proved to be safe and effective against cutaneous MCTs, squamous papilloma, and sebaceous gland adenoma in ferrets. Because of good effectiveness, low cost, and a relatively easy procedure ECT could represent a good treatment of choice instead of surgery for selected skin tumours in ferrets.

\section{Acknowledgment}

The authors would like to thank Dr Aleksandra Domanjko Petrič, Clinic for Small animals, Veterinary faculty, University of Ljubljana and Dr Nina Mlakar Hrženjak, Clinic for Birds, Small Mammals and Reptiles, Institute of Poultry, Birds, Small Mammals and Reptiles, Veterinary Faculty, University of Ljubljana, for performing abdominal ultrasound for staging of disease in ferrets.

\section{References}

1. Schoemaker NJ. Ferret oncology. Diseases, diagnostic, and therapeutics. In: Guzman DSM, editor. Veterinary Clinics of North America: exotic animal practice. Exotic animal oncology. Philadelphia: Elsevier; 2017. p. 183-208. doi: 10.1016/j.cvex.2016.07.004

2. Marini RP. Skin tumours. In: Mayer J, Donnelley TM, editors. Clinical veterinary advisor. Birds and exotic pets. St. Louis: Elsevier; 2013. p. 495-6.

3. Vilalta L, Meléndez-Lazo A, Doria G, Ramis A, Solano-Gallego L, Pastor J, et al. Clinical, cytological, histological and imunohistochemical features of cutaneous mast cell tumours in ferrets (Mustela putroius furo). J Comp Path 2016; 155: 346-55. doi: 10.1016/j.jcpa.2016.07.012

4. Orcutt C. Dermatologic diseases. In: Quesenberry KE, Carpenter JW, editors. Ferrets, rabbits and rodents. Clinical medicine and surgery. $2^{\text {nd }}$ edition. St. Louis: Saunders; 2004. p. 107-114.

5. Williams B. Neoplasia. In: Quesenberry KE, Carpenter JW, editors. Ferrets, rabbits and rodents. Clinical medicine and surgery. $2^{\text {nd }}$ edition. St. Louis: Saunders; 2004. p. 91-106

6. Rodrigues A, Gates L, Payne HR, Kiupel M, Mansell J. Multicentric squamous cell carcinoma in situ associated with papillomavirus in a ferret. Vet Path 2010; 47: 964-8. doi: 10.1177/0300985810369899

7. Beckwith-Cohen B, Teixeira LBC, Ramos-Vara JA, Dubielzig RR. Squamous papillomas of the conjunctiva in dogs: a condition not associated with papilomavirus infection. Vet Path 2015; 52: 676-80. doi: $10.1177 / 0300985814556185$ 
8. Cemazar M, Tamzali $Y$, Sersa G, Tozon N, Mir LM, Miklavcic D, et al Electrochemotherapy in veterinary oncology. J Vet Intern Med 2008; 22 : 826-31. doi: 10.1111/j.1939-1676.2008.0117.x

9. Johnson-Delaney CA. Ferret medicine and surgery. Washington: CRC Press; 2017.

10. Tozon N, Kodre $V$, Sersa G, Cemazar M. Effective treatment of perianal tumors in dogs with electrochemotherapy. Anticancer Res 2005; 25: 839-46.

11. Tozon N, Sersa G, Cemazar M. Electrochemotherapy: potentiation of local antitumor effectiveness of cisplatin in dogs and cats. Anticancer Res 2001; 21: $2483-6$.

12. Tozon N, Pavlin D, Sersa G, Dolinsek T, Cemazar M. Electrochemotherapy with intravenous bleomycin injection: an observational study in superficial squamous cell carcinoma in cats. J Fel Med Surg 2014; 16: 291-9. doi: $10.1177 / 1098612 \times 13507071$

13. Tozon N, Kramaric P, Kadunc Kos P, Sersa G, Cemazar M. Electrochemotherapy as a single or adjuvant treatment to surgery of cutaneous sarcoid tumours in horses: a 31-case retrospective study. Vet Rec 2016; 179: 627. doi: 10.1136/ vr.103867

14. Lanza A, Pettorali M, Baldi A, Spugnini EP. Surgery and electrochemotherapy treatment of incompletely excised mammary carcinoma in two male pet rats (Rattus norvegicus). J Vet Med Sci 2017; 79: 623-5. doi: 10.1292/ jvms.16-0578

15. Bruner CHM, Dutra G, Silva CB, Silviera GML, Martins MFM Electrochemotherapy for the treatment of fibropapillomas in Chelonia mydas. J of Zoo Wildl Med 2014; 45: 213-8.

16. Lanza A, Baldi A, Spugnini EP. Surgery and electrochemotherapy for the treatment of cutaneous squamous cell carcinoma in a yellow-bellied slider (Trachemys scripta scripta). J Am Vet Med Assoc 2015; 246: 455-7. doi: 10.2460/javma.246.4.455

17. Harrison TM, Kitchel BE. Principles and aplications of medical oncology in exotic animals. In: Guzman DSM, editor. Veterinary clinics of North America: exotic animal practice. Exotic animal oncology. Philadelphia: Elsevier; 2017. p. 209-34. doi: 10.1016/j.cvex.2016.07.007

18. Mayer J, Robat C. Lymphoma. In: Mayer J, Donelley TM, editors. Clinical veterinary advisor. Birds and exotic pets. St. Louis: Elsevier; 2013. p. 471-5.

19. Hamilton TA, Morrison WB. Bleomycin chemotherapy for metastatic squamous cell carcinoma in a ferret. J Am Vet Med Assoc 1991; 198: 107-8. 